\title{
LA DESCENTRALIZACIÓN DEL SISTEMA ESPAÑOL DE COMPETENCIA. VENTAJAS E INCONVENIENTES
}

\author{
Arseni Gibert Bosch
}

\begin{abstract}
Sumario: 1. Introducción 2. El Ámbito de las ventajas 3. El ÁmBITO DE LOS INCONVENIENTES 3.1. Algo sobre magnitudes 3.2. Algo sobre especialización 3.2.1. Especialización funcional en materia de enforcement 3.2.2. Especialización funcional en materia de advocacy 3.3. Algo sobre la proximidad 4. PARA FINALIZAR, UNAS CONSIDERACIONES SOBRE DOS TEMAS DE ACTUALIDAD RELACIONADOS CON LA DESCENTRALIZACIÓN.
\end{abstract}

\section{INTRODUCCIÓN}

No se puede decir que haya habido nunca unanimidad absoluta en relación a la conveniencia o no conveniencia de la descentralización del sistema español de defensa de la competencia propiciada por la Sentencia del Tribunal Constitucional (TC) de 11 de noviembre de 1999 (STC 208/1999)․․ La sentencia reconocía competencias ejecutivas a las Comunidades Autónomas (CCAA) en materia de defensa de la competencia en base a las ya consolidadas como propias de las mismas en materia de comercio interior y recogidas por sus Estatutos de Autonomía.

La STC 208/1999 fue aplicada o desarrollada mediante la Ley 1/2002, de 21 de febrero, de coordinación de las competencias del Estado y las comu-

${ }^{1}$ Dicha sentencia resolvió sendos recursos presentados por los gobiernos catalán y vasco a determinadas prescripciones de la Ley 16/1989, de 17 de julio, de defensa de la competencia (LDC-1989). 
nidades autónomas en materia de defensa de la competencia (Ley 1/2002) y en base a la misma, diferentes CCAA (no todas) y en diferentes momentos, principalmente entre los años 2003 y 2005, crearon sus primeros órganos autonómicos de competencia para el ejercicio de las competencias reconocidas.

Este proceso de descentralización fue inicialmente bastante controvertido $\mathrm{y}$, aunque la nueva situación se ha ido consolidando y aceptando de forma relativamente general, las reticencias latentes de los menos partidarios de la descentralización afloran intermitentemente de forma explícita. Se podría decir metafóricamente que a la manera del Guadiana.

Probablemente esta situación tiene su lógica a partir del hecho que, como casi todo, la descentralización tiene ventajas e inconvenientes. Trataré de unas y otros en este texto, intentando ser objetivo aunque no neutral, pues mi balance es claramente favorable a la descentralización, no tan solo casi por obligación en función de mi ubicación en el sistema, sino también por un convencimiento absoluto del mayor peso de las ventajas.

Con carácter previo al análisis sobre los beneficios o perjuicios del sistema, conviene mencionar a diversos aspectos que influyen o pueden influir en dicho análisis.

En primer lugar, hay que recordar que el modelo descentralizado era experimental y sigue siendo, en la Unión Europea (UE) prácticamente excepcional: solo en Alemania había ya un modelo descentralizado, con diferencias notables con el modelo español, pero con competencias significativas atribuidas a los Länder. En cambio, la aplicación del derecho de la competencia estaba y sigue estando centralizado en la mayoría de los estados miembros de la UE, salvo algún caso en que se ha otorgado alguna función auxiliar a las administraciones territoriales, como por ejemplo Italia, país en donde se ha iniciado, de hecho, una profundización en esta línea descentralizadora.

Esta clara mayoría puede hacer pensar en la no necesidad o conveniencia de descentralizar y situar por ello el modelo descentralizado en una variable del campo de los inconvenientes. Sin embargo, algunos episodios concretos más allá de la UE pueden suscitar percepciones de signo contrario. Un ejemplo claro y además muy famoso es el caso Microsoft en los EEUU. La Federal Trade Comission (FTC) actuó impelida por la acumulación de hasta 17 denuncias o quejas procedentes de organismos de competencia o similares de 17 estados de la Unión. Más adelante, al hablar de las ventajas, veremos cómo en España, las estadísticas son favorables a la descentralización puesto que, empíricamente, acreditan que tanto la actividad sancionadora como las actuaciones pro competitivas de otra naturaleza se han ampliado con la descentralización y han abarcado supuestos o subsectores no abordados anteriormente con el modelo centralizado. 
Además, la diversidad de modelos estructurales de los órganos de defensa y promoción de la competencia en el mundo, no se limita a la dicotomía centralizado/descentralizado dentro de cada estado. Ni siquiera es esta la diferencia más relevante. Hagamos un pequeño inciso para desviarnos, por un breve espacio del texto, del tema central y veamos una breve descripción panorámica de otras diferencias de modelo.

Hay casos en los que solo se ejerce la función sancionadora (enforcement) y prácticamente no se actúa en el campo que en España hemos dado en llamar promoción de la competencia (advocacy). De hecho así era en buena medida en la Europa continental hasta que la Comisión Europea en su Comunicación «Una política de Competencia Pro-activa para una Europa Competitiva» de 20 de abril de $2004^{2}$, que formaba parte del nuevo paquete de modernización de las normas comunitarias de competencia, dio un fuerte impulso a las políticas de promoción de la competencia. En España los primeros informes sobre la situación de no competencia de algunos sectores se hicieron públicos durante los años 90. Eran trabajos descriptivos sobre la escasa o nula competencia en algunos sectores importantes de la economía, sugerían remedios para introducirla y consiguieron un cierto impacto por su novedad en el panorama del análisis de la situación económica. La emisión de algunos informes de ese tipo continuó a partir de la Ley 16/1989, de 17 de julio, de defensa de la competencia (LDC-1989) que no ponía énfasis en la promoción de la competencia. Las recomendaciones de esos informes fueron por lo general poco atendidas por los sucesivos gobiernos, una situación que ha mejorado algo a lo largo de los años aunque no de forma muy significativa.

Pero hay más e importantes diferencias entre modelos. Una muy relevante es la relativa a las condiciones y garantías de independencia de los organismos. Hay casos explícitos de no independencia, es decir, organismos que son directamente unidades administrativas de los gobiernos, aunque lo más frecuente es que sean independientes, tanto respecto de los operadores económicos como de los gobiernos. Una independencia necesaria, esencial para ser más exactos ${ }^{3}$. Pero incluso entre los no independientes hay enormes diferencias: en Australia y Nueva Zelanda, por ejemplo, el grado de independencia decisoria es elevado aunque instrumentado de forma distinta a la habitual: la función instructora se ejerce directamente por parte de unidades gubernamentales pero resuelve la jurisdicción mediante tribunales especializados en competencia que, además, controlan la calidad pro competitiva de la regulación ${ }^{4}$.

${ }^{2}$ http://eur-lex.europa.eu/procedure/ES/190522.

${ }^{3}$ Para constatarlo solo hay que observar algunas reacciones de los departamentos gubernamentales impulsores de normas sectoriales ante informes críticos de los órganos de competencia.

${ }^{4}$ Algo poco necesario en esos países. 
En España, hasta el año 2007 el órgano instructor era una unidad administrativa del Ministerio de Economía y el órgano independiente solamente resolvía y emitía algunos informes. La propia Comisión Europea tiene una Dirección General de Competencia que resuelve directamente los expedientes, aunque están sometidos a la posterior revisión por parte del Tribunal de Justicia de la Unión Europea (TJUE). Son excepciones que confirman la regla general de independencia cuando menos formal, puesto que la efectiva depende en buena medida de la consolidación en cada país de la cultura de independencia de las instituciones que la requieren ${ }^{5}$. En última instancia, lógicamente, lo decisivo es la independencia de ejercicio, es decir, como casi siempre en asuntos de esa índole, lo decisivo son las personas.

Finalmente, en el campo de los modelos, el más habitual es el especializado estrictamente en competencia. Así era en España hasta 2013. Pero algunos abarcan otras funciones: regulación sectorial de sectores específicos, políticas de defensa de los consumidores y usuarios etc ${ }^{6}$.

\section{EL ÁMBITO DE LOS INCONVENIENTES}

Recuperando el núcleo central del texto, se intenta ahora una aproximación a los principales inconvenientes.

Tal vez el más esgrimido por los poco entusiastas de la descentralización sea el relativo a los riesgos de aplicación no homogénea de las normas de competencia. Sin negar el hecho de que cuántos más organismos apliquen las mismas normas más posibilidades de incremento de un riesgo que conviene evitar, por varias razones este temor me parece una apreciación bastante subjetiva. En el sistema español, en principio horizontal, sin relación jerárquica entre el organismo central y los autonómicos, se establece sin embargo una

5 Tomemos como referencia nuestro país. Cuando un organismo independiente (excluyo ahora los de competencia ya que, por diversas razones de carácter positivo entre las cuales que sus resoluciones finalizan la vía administrativa pero están sometidas a la revisión jurisdiccional, tienen una tradición mejor que otros organismos). Pero en general es fácil observar que, con frecuencia, el día anterior al previsto para la toma de una decisión importante por parte de alguno de estos organismos, los medios de comunicación nos muestran una mesa con la ubicación en la mismo de los miembros - con sus nombres-del órgano colegiado del organismo en cuestión y con indicación del partido que los ha propuesto y en base a ello pronostican el resultado de la votación. Casi siempre aciertan.

${ }^{6}$ En el caso español, la actual Comisión Nacional de los Mercados y la Competencia (CNMC) tiene una cierta similitud con el organismo holandés y con el lituano, integra competencia, la regulación de siete sectores y tiene algunas funciones relacionadas con la aplicación de la Ley 20/2013, de 9 diciembre, de Garantía de la Unidad de Mercado. Algunos países han integrado reguladores sectoriales pero han mantenido separado el de competencia, es el caso de Alemania con el Bundeskartellamt. 
cierta relación de primacía del central en diversos aspectos. Uno de ellos, tal vez el más importante, es la capacidad del órgano central para personarse como parte interesada en los expedientes que tramitan los órganos autonómicos de competencia, lo que le permite alegar en los procedimientos contradictorios en la fase de instrucción y también, lo que es más importante y a mi juicio impropio o cuando menos mal instrumentado, impugnar resoluciones de dichos órganos autonómicos de competencia ${ }^{7}$.

La descentralización no es ni el único ni el más importante factor de riesgo. En este sentido, basta con analizar resoluciones de un mismo órgano, tanto central como autonómico, para comprobar que, sobretodo según las distintas etapas y composición de los mismos, tampoco resulta muy difícil constatar criterios distintos e incluso contradictorios. Y eso es así en todos los niveles, autonómico, central de todos los estados miembros e incluso europeo en aplicación de los artículos 101 y 102 del Tratado de Funcionamiento de la Unión Europea (TFUE). Afortunadamente, en todos los casos el intérprete final de las resoluciones es la jurisdicción correspondiente en cada supuesto ${ }^{8} \mathrm{y}$ en las distintas instancias a las que las partes pueden apelar. Y es entonces cuando acaba la discusión y queda sentada jurisprudencia que, como es inevitable, tampoco siempre es perfectamente uniforme. En resumen, podríamos afirmar que, el garante de la aplicación correcta y homogénea de las normas no es otra, en realidad, que la jurisdicción.

Por supuesto que los órganos autonómicos creemos que hay que esforzarse permanentemente para que dicha aplicación sea lo más homogénea posible, pero son pertinentes dos consideraciones más. La primera es que la im-

${ }^{7}$ Una facultad que hasta 2007 estaba atribuida al Servicio de Defensa de la Competencia (SDC), una unidad administrativa del Ministerio de Economía que instruía los expedientes de infracción y los trasladaba al Tribunal de Defensa de la Competencia (TDC), órgano independiente de resolución que en principio debiera ser el que, en caso de existir esta facultad, debiera ejercerla. Esta disfunción se trasladó por vía de disposiciones adicionales genéricas de atribución de todas las funciones del SDC a los órganos de instrucción/ investigación de las nuevas instituciones de competencia ya integradoras de todas las funciones, en 2007 la Comisión Nacional de la Competencia (CNC) y en 2013 la CNMC y no fue atribuida como parecería lógico a los órganos de resolución de dichas nuevas instituciones. Parece plausible poner en duda si la intención expresa del legislador era la de excepcionar esta función de la prescripción del art.8f del Real Decreto 657/2013, de 30 de agosto, por el que se aprueba el Estatuto Orgánico de la Comisión Nacional de los Mercados y la Competencia, que, al asignar al Consejo de la CNMC (órgano resolutorio) sus funciones reza como sigue: Acordar, a propuesta del Secretario del Consejo o de otras unidades competentes de la Comisión, la interposición de recursos, la personación en procesos judiciales y procedimientos administrativos y la intervención, como amicus curiae, en los procedimientos a que hace referencia la Ley 1/2000, de 7 de enero, de Enjuiciamiento Civil.

8 Tribunales Superiores de Justica de las CCAA, Audiencia Nacional, Tribunal Supremo o equivalentes en todos los países y TJUE a nivel europeo. 
pugnación no parece el único ni el mejor instrumento para coordinar la aplicación homogénea de las normas. La segunda, que en cualquier supuesto -parece una obviedad- los instrumentos de dicha coordinación deberían ser entre órganos de instrucción en fase de instrucción y entre órganos de resolución en fase de resolución. En ningún caso que un órgano de instrucción «vigile» órganos de resolución.

Otro inconveniente de la descentralización es cierta dificultad de aplicación de la Ley 1/2002 en diversos aspectos, tal vez el principal el relacionado con la asignación de casos entre el órgano central y los autonómicos, fundamentalmente regido por los puntos de conexión definidos por la citada Ley y cuyo criterio básico es el de los efectos sobre un territorio que desborde o no el de la respectiva comunidad autónoma. Admitiendo de entrada que la delimitación no puede ser perfecta y que por lo tanto el problema no es soluble al $100 \%$, mi opinión personal, ya expresada frecuentemente por especialistas en este tipo de materias, que no es mi caso, es que la Ley 1/2002 debería haber sido integrada en la nueva Ley 15/2007, de 3 de julio, de defensa de las competencia (LDC2007). De este modo, se habría aprovechado la oportunidad para mejorar las disfunciones detectadas y resolver los puntos débiles, puestos en evidencia por la experiencia en su aplicación y que dan pie a interpretaciones excesivamente discrecionales que generan algunos conflictos. Hay que reconocer que la buena voluntad de las partes ha evitado que dichos conflictos proliferen y se agraven; asimismo, esta buena voluntad por parte de todos, se ha traducido en un conjunto de mecanismos que se dieron en llamar "colaboración no reglada» que permiten, sin forzar en ningún caso la norma, usar los márgenes de diálogo, intercambio ágil de información y flexibilidad en la interpretación cuando esta es posible, que ha dado y está dando muchos frutos y evitando muchos conflictos elevados al mecanismo de arbitraje reglado previsto'. Unos buenos resultados lógicamente modulados con el tiempo, según los interlocutores cambiantes responsables de la utilización de dichos instrumentos.

Vinculado al mecanismo de asignación de casos, la descentralización presenta otro potencial inconveniente, el riesgo de fórum shopping, es decir, que un presunto infractor tenga medios y oportunidad de elegir la autoridad de competencia que tramitará el caso. El riesgo es mínimo y tiende a cero si no concurre el consentimiento o la negligencia de alguna de las autoridades. Sin embargo, no podemos asegurar que no se haya dado algún caso de forum shopping durante los años transcurridos desde el inicio del proceso de descentralización, pero si se ha producido, ha sido en contadas ocasiones. A estos efectos es importante minimizar los casos de traslado de expediente y

${ }^{9}$ Lo cual supone evitar generar y dilucidar conflictos formales y, sobretodo consumo de tiempo en los procedimientos, un tiempo que en ocasiones, cuando se alarga, puede ir en detrimento de los intereses de alguna de las partes implicadas en el procedimiento. 
cuando sea necesario que se produzcan, tener claras las reglas y el control para eludir este riesgo.

Por último, otro riesgo subyacente es el de la distancia y el tamaño de los órganos de competencia. De la misma manera que los reguladores de los gobiernos locales tal vez sean más susceptibles de ser convencidos, o capturados, por los operadores económicos en los distintos mercados, que los reguladores de los gobiernos autonómicos, e igual suceda con éstos en relación a un gobierno central más distante y de más entidad, no se puede despreciar el riesgo de a que los órganos autonómicos de competencia les ocurra algo parecido en relación con el central. Pero no hay que sobrevalorar este riesgo. Los tamaños y capacidad de presión de las empresas y los lobbys son también mayores, en alguna medida proporcionalmente a los tamaños y capacidad de resistencia de los gobiernos de cada nivel y los órganos autonómicos en relación al central. En cualquier caso, además de la eventual presión de los operadores, los gobiernos - de todos los niveles- también caen algunas veces en la tentación de intentar influir en las decisiones de los organismos de competencia. Una vez más hay que insistir en el hecho que, en última instancia todo depende de la independencia de ejercicio, y ésta depende principalmente de las personas.

Y creo sinceramente que aquí se puede cerrar el capítulo de inconvenientes.

\section{EL ÁMBITO DE LAS VENTAJAS}

Por lo que se refiere a las ventajas de la descentralización, éstas pueden dividirse en tres: 1) de MAGNITUD; 2) de ESPECIALIZACIÓN; y 3) de PROXIMIDAD.

\subsection{Algo sobre magnitudes}

Si tuviéramos alguna certeza de que los problemas de competencia que detectamos, investigamos, tramitamos y resolvemos mediante procedimientos sancionadores o que tratamos desde los distintos tipos de documentos y de puntos de vista de los trabajos de promoción de la competencia, tienen una correlación más o menos aproximada con el universo de problemas de mal funcionamiento de los mercados por razón de infracciones o disfunciones derivadas de insuficiente competencia por diversas razones, tal vez la descentralización aportaría relativamente pocas ventajas.

Pero no podemos estar nada seguros de que sea así, es posible o más bien probable que la cantidad oculta de problemas tenga una magnitud extraordinariamente mayor que la parte conocida. Aplicando una sencilla metáfora, puede que la parte conocida (incluida la intuida) sea el agua de los ríos y los lagos de la tierra, y que la parte no conocida sea la de la inmensidad oceánica. 
Intentando una aproximación que pretende ser conceptualmente descriptiva tal vez sean procedentes algunas preguntas:

- ¿Cuántos cárteles hay?

- ¿Cuántas conductas ilícitas en términos de competencia se producen?

- ¿Qué relación hay entre estas magnitudes y las de los casos detectados y tramitados por los órganos de competencia?

Son preguntas sin respuesta. Si se detectan 50 cárteles podemos pensar que hay 100 , si detectamos 101 , podemos pensar que hay 500, si detectamos 501 podemos pensar que hay 1000 . Algo parecido ocurre con las prácticas ilícitas. Si se denuncian o se detectan 100 podemos pensar que hay $500 \mathrm{o}$ directamente 1000, o 2000, o más.

Lo que sí sabemos es que se han detectado casi por casualidad cárteles poco previsibles que han venido siendo operativos durante 25 años o más. Lo que también sabemos es que cada vez que, por las razones que sea, investigamos o estudiamos un nuevo sector o subsector, casi siempre, por no decir siempre, llegamos a la conclusión de que hay no solo el problema de competencia del que habíamos tenido indicios, sino también alguno más. Y asimismo sabemos que los problemas que se detectan en un territorio, con toda probabilidad, idénticos o parecidos, se producen también en otros territorios.

Seguimos sin saber la magnitud de lo que no se detecta, pero lo que sabemos permite afirmar sin ningún género de dudas que se trata de una magnitud muy grande, probablemente enorme.

Hasta aquí una constatación de lo que ignoramos que ocurre en una parte del trabajo de los órganos de competencia, la que viene determinada por el enforcement, por las prácticas anticompetitivas sancionables del ámbito empresarial y de las administraciones cuando actúan como operadores económicos. Es decir, la parte vinculada al procedimiento sancionador que tal vez podríamos estimar, aunque obviamente sin precisión, como aproximadamente la mitad del problema global.

De un problema que tiene su lógica, puesto que las empresas vivirían mejor sin competencia, por lo menos a corto plazo: ganarían más dinero sin necesidad de competir y a costa de otras empresas o de los consumidores y usuarios de bienes y servicios. A largo plazo seguramente les iría peor, perderían temple competitivo, la economía de su entorno se debilitaría y perdería paulatinamente su capacidad de competir con empresas operativas en entornos más habituados a la competencia. Ello ha sido siempre así y lo es todavía mucho más en un mundo globalizado y con decrecientes barreras territoriales de tipo proteccionista. Pero el razonamiento básico en muchas ocasiones se centra en el corto y el medio plazo y en realidad los cárteles son muy productivos desde este punto de vista. 
Hemos afirmado que esto es algo parecido a la mitad del problema. Pasemos ahora a la otra mitad con algunas nuevas preguntas:

- ¿Cuántas normas hay?

- ¿Cuántas de ellas contienen prescripciones anticompetitivas no justificadas, no proporcionales y no discriminatorias?

- ¿Qué relación hay entre las que informamos y sobre las cuales hacemos recomendaciones y el número global de normas existentes?

Para esas preguntas no tenemos tampoco respuestas o las tenemos solo muy parciales. Sabemos que en España hay un gobierno central y el número de Ministerios que el mismo tiene en cada momento. Sabemos que hay 17 gobiernos autonómicos con su número de consejerías asimismo variable en el tiempo. Sabemos que cada uno de estos ámbitos tiene sus cámaras legislativas, cuyos grupos parlamentarios tienden a priorizar las enmiendas de adición y a olvidar que existen las de supresión. Sabemos que hay cincuenta diputaciones provinciales, consejos comarcales en algunas CCAA, alrededor de 9000 ayuntamientos y algunas otras entidades locales. Y solo tenemos una idea bastante remota de cuántos organismos relacionados con estas administraciones (consorcios, mancomunidades, empresas, fundaciones, organismos autónomos de diversos tipos, medios propios de todas esas administraciones con distintas forma de personalidad jurídica etc.).

Lo que no sabemos es cuántas normas de todo rango (leyes, decretos, ordenes, otras disposiciones, ordenanzas municipales diversas etc.) están vigentes y se promulgan cada año. Por lo tanto no conocemos sus contenidos eventualmente anticompetitivos, son demasiadas para poder leerlas, haría falta un impensable número de lectores cualificados y un número todavía mayor de personas cualificadas para actuar sobre los problemas detectados.

Tampoco sabemos en cuántas ocasiones -todos estos agentes- actúan como operadores económicos y lo hacen con orientación anticompetitiva. Ni tampoco el número, ni siquiera aproximado, de encomiendas de gestión que se encargan mutuamente para evitar licitaciones que deberían hacerse. No sabemos la cantidad licitaciones para obras, concesiones, y contratación pública generan todas estas entidades, aunque sabemos que es un número ingente, enorme. No sabemos cuántas de estas licitaciones están en alguna medida pre adjudicadas ni las que, consciente o inconscientemente, facilitan la colusión por parte de las empresas que concurren. Tampoco sabemos cuántas ayudas públicas no notificables a Bruselas gestionan y que, pese a ser menores, pueden también distorsionar la competencia. Solo conocemos algunas de las rentas regulatorias derivadas del clientelismo de algunas administraciones. 
Es mucho lo que no sabemos, muchísimo más que lo que sí sabemos. Porque sobre esta inmensa cantidad de normas, actos, decisiones, licitaciones, ayudas, etc., casi lo único que sabemos con cierta seguridad, por la experiencia en forma de muestra derivada de nuestro trabajo sobre estos temas, es que se trata de una magnitud que puede suponer aproximadamente la otra mitad del problema. Tal vez más.

Porque en realidad lo que ignoramos no es solo lo que pasa, dónde y cuándo pasa. Lo más importante es que ignoramos el potencial real existente de mejora de la eficiencia de los sistemas económicos y de los beneficios para consumidores y usuarios en supuestos de optimización de las condiciones de competencia.

$\mathrm{Y}$ es a partir de esta enorme bolsa de desconocimiento que deducimos una de las grandes ventajas de la descentralización, que no es otra que el incremento de agentes pro competitivos y, sobretodo, el incremento de actividad tanto en enforcement como en advocacy que ha producido la descentralización incremento de detección de casos, incremento del número de expedientes sancionadores en alguna medida por el factor proximidad, de normas revisadas y de sectores o subsectores analizados, de propuestas y recomendaciones sobre la mejora pro competitiva de la regulación. Un incremento modesto, sin duda, pero modesto era también el punto de partida.

Para confirmar este incremento nada mejor que su cuantificación, tanto en enforcement como en advocacy, aunque sea con alguna imprecisión ${ }^{10}$. Partiendo de los datos de $2002^{11}$, se han registrado por bienios y hasta 2013, las actividades acumuladas de los órganos de competencia (central y autonómicos en sus distintas estructuras en diversas etapas temporales).

Veamos como resumen de trabajo de recopilación efectuado ${ }^{12}$ los respectivos gráficos de ambos tipos de actividad.

${ }^{10}$ Imprecisión inevitable, sobretodo en advocacy por las diferentes nomenclaturas de las distintas autoridades sobre los trabajos realizados, por algunas pequeñas discrepancias entre memorias y páginas web, por no computación de trabajos menores o simplemente no registrados etc.

11 Antes de la descentralización dado que los primeros trabajos de los primeros órganos autonómicos de competencia creados datan de 2003.

${ }_{12}$ En la ACCO están disponibles los datos sintetizados en estos dos gráficos clasificados por tipologías (con desglose detallado) y por órgano emisor. Se ha considerado que a efectos globales y dada la relativa imprecisión ya reconocida anteriormente, lo importante era incluir en este texto una imagen clara de la intensidad del crecimiento de actividad global, en buena medida debida a la descentralización. 


\section{Gráfico 1. Datos agregados relativos a las resoluciones en materia de de- fensa de la competencia adoptadas por el conjunto de organis- mos de competencia ${ }^{13}$}

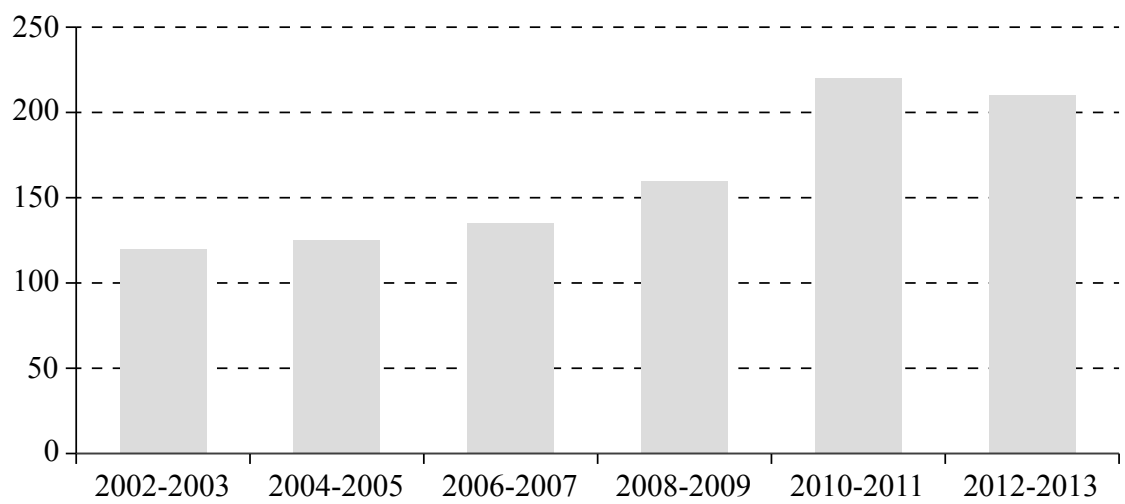

\section{Gráfico 2. Datos agregados relativos a los documentos de promoción ela-} borados por el conjunto de organismos de competencia ${ }^{14}$

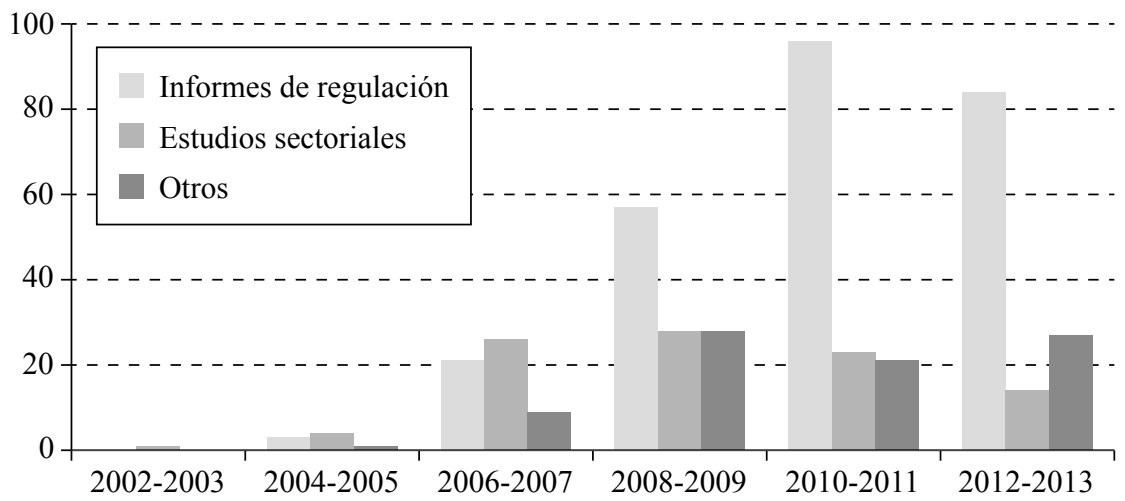

${ }^{13}$ Los datos contenidos en este gráfico son datos aproximados obtenidos a partir de la consulta de las páginas web de los distintos organismos de competencia (estatal y autonómicos) así como de sus respectivas memorias de actividades. Incluyen las resoluciones recaídas en expedientes sancionadores, archivos, terminaciones convencionales y resoluciones de recursos.

${ }^{14}$ Los datos contenidos en este gráfico son datos aproximados obtenidos a partir de la consulta de las páginas web de los distintos organismos de competencia (estatal y autonómicos) así como de sus respectivas memorias de actividades. No se han tenido en cuenta 
Los datos hablan por si solos: el incremento de agentes, a pesar de su modestia y escasez de medios en muchos casos, ha supuesto un incremento de actividad relativamente notable en el ámbito del enforcement (el factor proximidad ha incidido en denuncias que antes no se concretaban) y muy notable, casi se podría decir espectacular, en advocacy, en cantidad de normas informadas, en estudio de mercados locales, etc. Aunque hay que tener en cuenta que en este ámbito una parte importante de este crecimiento corresponde al órgano central, pues es a partir de 2007, con la nueva LDC, que el concepto de promoción de la competencia se incorpora en el ámbito de las funciones obligatorias de los órganos de competencia, así como también se configura como obligatoria la petición de informe de regulación sobre normas con rango de Ley.

Y aún algo más importante: el potencial de crecimiento de actividad global derivada de la descentralización es mucho mayor del actualmente efectivo. En estos momentos tan solo hay 4 órganos autonómicos de competencia que, a pesar de su limitación de recursos materiales y humanos, pueden considerarse «completos», es decir, que por una parte instruyen y resuelven y, por otra, realizan una actividad de promoción de la competencia de magnitud significativa. El resto tienen estructuras muy diversas, algunos solo instruyen expedientes y su característica común es su pequeña dimensión y escasez de medios. Hay que añadir que algunas CCAA no han creado ningún tipo de estructura y que alguna otra lo ha hecho muy recientemente. Un desarrollo de la descentralización más completo y homogéneo, aún sin grandes estructuras ni medios, desarrollaría este potencial a niveles mucho más elevados.

\subsection{Algo sobre especialización}

Las actuaciones de las autoridades de competencia son diversas y de naturaleza bastante diferenciada. Algunas más propias de un organismo central

los datos relativos a los informes sobre ayudas públicas, informes sobre concentraciones y los antiguos informes de apertura de establecimientos comerciales.

Según los datos expuestos se puede constatar un aumento significativo del número de documentos de promoción elaborados a partir de la entrada en vigor del nuevo esquema institucional y la creación de los organismos autonómicos de competencia. En cuanto al periodo 2010-2011 es relevante el gran número de informes de regulación elaborados, los cuales responden, en gran parte, a la actividad de adaptación normativa a la Directiva 2006/123/CE del Parlamento europeo y del Consejo, de 12 de diciembre de 2006, relativa a los servicios en el mercado interior (Directiva de Servicio). Asimismo, la ligera disminución en la actividad de promoción de la competencia durante el período 2012-2013 puede deberse, en parte, tanto a la supresión de algunos órganos autonómicos como a la disminución de medios materiales y principalmente humanos que haya podido producirse como consecuencia de los ajustes de personal efectuados por las Administraciones Públicas durante los últimos años. 
potente en estructura y medios, otras más asequibles para órganos más flexibles y ágiles para que sus técnicos alternen con facilidad actividades distintas. También en este sentido la descentralización ofrece algunas importantes ventajas.

\subsubsection{Especialización funcional en materia de enforcement}

Un primer nivel de especialización comporta que el organismo central sea el idóneo para instruir y resolver los casos más importantes por multitud de razones: la magnitud de los efectos; el ámbito territorial afectado; la necesidad de aplicación de medidas disuasorias a grandes empresas con medios propios o profesionales de defensa solo equiparables a los medios disponibles en un organismo de mayor tamaño, más entidad y recursos de inspección (informática sofisticada, inspecciones simultáneas que requieren mucho personal ${ }^{15}$ etc.) que los disponibles por parte de los órganos autonómicos de competencia, etc. También para la aplicación de los programas llamados de «clemencia», puesto que los grandes cárteles suelen actuar en un ámbito territorial que desborda el de una comunidad autónoma, y requieren asimismo capacidades de gestión de este tipo de casos más adecuadas que las habituales disponibles en los órganos autonómicos de competencia.

Sin duda, la liberación del órgano central del deber de instruir y resolver casos de menor entidad, favorece este tipo de especialización y redunda en mayor eficacia para detectar, investigar conductas de grandes operadores y disuadir y restaurar las condiciones de mercado en sectores de gran importancia para la eficiencia económica y afectación a gran número de consumidores y usuarios

Los órganos autonómicos de competencia, lógicamente especializados en casos con afectación menos extensa territorialmente y con efectos adversos para menos personas o empresas, terminan conociendo mejor las especificidades y los casos que se replican a lo largo de su territorio. En este sentido, reciben inputs desde ámbitos próximos y la detección de algunos tipos de problemas tiende a ser más probable.

\subsubsection{Especialización funcional en materia de advocacy}

En un sentido muy equiparable al expuesto en el punto 2.1, también los trabajos de promoción de la competencia admiten una clasificación por trascendencia, efectos prácticos e importancia de la afectación en los mercados

${ }^{15}$ Es justo destacar que cuando los órganos autonómicos de competencia han necesitado refuerzos de este tipo para alguna inspección, el órgano central siempre ha respondido satisfactoriamente y destacar, asimismo, que dichos órganos también han colaborado frecuentemente con inspecciones del órgano central en los respectivos territorios. 
de las recomendaciones de los trabajos, tanto si se refieren a la regulación como al funcionamiento de los mercados sectoriales o subsectoriales.

En el caso de la regulación es fácil formular dos afirmaciones: (a) en los casos -que los hay- en los que la calidad de la legislación estatal es alta, principalmente la de carácter básico o la que regula las instituciones locales, esta calidad debería trasladarse en cascada a las regulaciones sectoriales respectivas de los otros niveles de la administración pero no siempre sucede así; y (b) es impensable que un órgano central, por muchos medios que disponga, pueda llegar a detectar problemas de competencia derivados, poniendo un ejemplo extremo, de ordenanzas municipales. Y ya hemos dicho y está comprobado que los hay en abundancia. No se trata de que los órganos autonómicos lleguen a todas partes (por lo general, y debido a sus medios relativamente precarios, poco trabajo de promoción de la competencia pueden hacer en momentos de puntas de trabajo en casos de instrucción de expedientes sancionadores), pero sin duda sus recursos los pueden aplicar, y los aplican en buena medida, a aspectos que quedan muy lejos de los ámbitos más globales de actuación del organismo central.

Esa complementariedad se manifiesta también en la cooperación entre el órgano central que, al elaborar estudios sectoriales puede contar, y de hecho cuenta, con la colaboración de los órganos autonómicos aportando información específica de sus territorios respectivos.

\subsection{Algo sobre la PROXIMIDAD}

Ya ha quedado dicho que la proximidad puede ayudar a la detección de problemas específicos. Pero su verdadera dimensión como ventaja de la descentralización es en la mayor penetración potencial de lo que hemos convenido en llamar «cultura de competencia». Hay que insistir: potencial. No es que sea fácil, puesto que incrementar la cultura de competencia requiere tenacidad y paciencia. Todavía algunas empresas, sobretodo pequeñas, o sus organizaciones más o menos gremiales acuden a los órganos autonómicos con intención de denunciar por competencia desleal a algún competidor que según ellos «revienta precios»y debemos explicarles, no sin alguna dificultad, la naturaleza de la competencia y sus virtudes. Asimismo, todavía muchísimos consumidores y usuarios no tienen una noción muy clara de las ventajas que les suponen la introducción o el incremento de competencia en los distintos sectores.

Aunque por otra parte, la experiencia de estos años acaba demostrando que el trabajo de promoción no suele tener efectos inmediatos o espectaculares en ninguno de sus objetivos, pero acaban penetrando y ofreciendo resultados. Ya ha ocurrido en alguna ocasión que los grupos parlamentarios de las parlamentos y asambleas legislativas autonómicas han usado informes de regulación de los órganos autonómicos de competencia para pedir explica- 
ciones al respectivo gobierno. También hay casos de informes de regulación que no han sido tomados en consideración por los departamentos del gobierno impulsores de las normas informadas, pero en cambio sí han contribuido a mejorar la regulación sectorial cuando algunos años después han sido modificadas por otras razones. Hay que insistir: paciencia y tenacidad.

Por otra parte hay algo muy importante que hay que tomar en consideración y valorar adecuadamente. Se trata del crecimiento considerable y constante de las consultas que se reciben en los órganos autonómicos de competencia. Consultas que realizan municipios, colegios profesionales, asociaciones gremiales, empresas y particulares. Consultas que son, por supuesto, siempre contestadas, en ocasiones verbalmente y más frecuentemente por escrito. Un trabajo de los órganos autonómicos que aunque se registra, no ha sido computado en los datos sobre actividad reflejados en los gráficos 1 y 2 , excepto en los casos en que, por tener un interés más general, se realizan versiones genéricas y son hechas públicas.

En ocasiones la respuesta es delicada. Hay que poner atención en no incurrir en el riesgo de generar un supuesto que pueda dar pie a una apelación a la confianza legítima, y para ello hay que tender a dar respuestas más explícitas en relación a «lo que no se puede hacer» que a «lo que sí se puede hacer». Pero, en cualquier caso, este es un campo de generación de cultura de competencia de magnitud creciente y que por ello está adquiriendo una notable importancia.

\section{PARA FINALIZAR, UNAS CONSIDERACIONES SOBRE DOS TEMAS DE ACTUALIDAD RELACIONADOS CON LA DESCENTRALIZACIÓN.}

Insistiendo en la importancia de la cultura de competencia, tal vez sea oportuno mencionar, la extraordinaria oportunidad que ofrece la transposición de la Directiva 2014/104/UE del Parlamento Europeo y del Consejo de 26 de noviembre de 2014 relativa a determinadas normas por las que se rigen las acciones por daños en virtud del Derecho nacional, por infracciones del Derecho de la competencia de los Estados miembros y de la Unión Europea (Directiva 2014/104/UE). Hay un aspecto de la misma que tiene el máximo interés desde el punto de vista de la promoción de la cultura competencia: la posibilidad de reclamación colectiva, incluso cuando los perjudicados son personas indeterminadas o de difícil determinación. Tal posibilidad figuró durante mucho tiempo en el texto del proyecto de Directiva pero se fue diluyendo durante las negociaciones, de manera que finalmente no se incluyó como obligación en el texto de la Directiva 2014/104/UE.

Sería importante regular esta posibilidad de tal forma que resultara viable dicho tipo de reclamaciones, lo cual es sin duda posible puesto que por lo menos en un Estado miembro -el Reino Unido- pero también aunque algo 
menos en Alemania, Dinamarca u Holanda, este tipo de reclamaciones se produce con cierta frecuencia.

A mi juicio, tal decisión comportaría tres importantes ventajas para las políticas de consumo (muy descentralizadas) y de competencia:

a) En primer lugar, las asociaciones de consumidores y usuarios tendrían una fuente de financiación que las reforzaría sensiblemente, lo que permitiría eliminar las subvenciones que reciben y sus consecuencias en forma de dependencia de la administración, variable que afecta sin duda a las políticas de consumo en relación con servicios públicos asimismo muy descentralizados tales como los sanitarios, educativos, de transporte público y otros.

b) Por otro lado, se eliminaría el tratamiento individual -caso a caso-de cada una de las reclamaciones de consumo que se presentan, de forma muy repetitiva, por un elevado número de usuarios y principalmente relacionadas con algunos servicios públicos de interés general prestados por empresas privadas ${ }^{16}$. Se trata de supuestos en los que suele haber algún problema de competencia. Tratar individualmente estas reclamaciones tiene dos graves inconvenientes: a) es muy costoso para la administración responsable de la defensa de los consumidores y usuarios, y b) no es disuasorio (vía sanción o vía mediación), ya que se resuelven reclamaciones individuales de la minoría reclamante pero la práctica que motiva la reclamación se sigue produciendo a costa de la mayoría no reclamante. Trasladar estos casos a las políticas de competencia permitiría efectos más disuasorios tanto desde el punto de vista de la sanción directa como de la reclamación posterior de daños que se propone regular.

c) Finalmente, la advocacy, o promoción de la competencia, recibiría un impulso importantísimo debido a que algunos de estos casos de reclamaciones colectivas resultarían muy notorios y producirían un eficaz paso adelante en la percepción de los ciudadanos respecto a cómo las restricciones a la competencia les afectan directamente.

Por otro lado, está irrumpiendo con fuerza un fenómeno de matriz tecnológica, y con un desarrollo y crecimiento vertiginosos, un fenómeno que, de forma probablemente provisional y con discutible acierto, se ha convenido en llamar economía colaborativa. No lo vamos a abordar en profundidad por varias razones; plantea muchas incertidumbres sobre las que reflexionar, entra en conflicto con sectores tradicionales lo que acentúa pulsiones proteccionistas y resistencias al cambio a pesar de la conciencia generalizada de que es impara-

${ }^{16}$ Un tipo de reclamaciones masivas que en el Reino Unido denominan «supercomplaints». 
ble e introduce elementos novedosos ${ }^{17}$ que requieren probablemente flexibilidad y cambios significativos en la cultura regulatoria más convencional.

Si se menciona en este texto es porque por una parte, tiene claras implicaciones con la competencia y por otra, se desarrolla paradójicamente en un ámbito conceptualmente global, pero con aplicación concreta en ámbitos locales en muchos casos; es decir, relaciona temática de competencia con descentralización del sistema. La ACCO publicó en julio de 2014 un documento de carácter más bien descriptivo ${ }^{18}$ y sigue trabajando para profundizar en el seguimiento y comprensión del fenómeno. Por otra parte la Dirección de Promoción de la Competencia de la CNMC está elaborando un estudio sobre dos sectores concretos concernidos por la económica colaborativa. La evolución de este fenómeno estará, sin duda, muy presente en los próximos años en la actividad de los órganos de competencia.

A modo de conclusión brevísima, cabe esperar que este texto haya contribuido modestamente a la valoración positiva del modelo de competencia descentralizado.

TITTLE: The Decentralization of the Spanish System of Competition. Advantages and disadvantages.

RESUMEN: En las presentes lineas se aborda con la necesaria 'altura de miras' tanto los potenciales inconvenientes como las irrefutables bondades de la descentralización del sistema español de defensa de la competencia. Desde el prisma de los inconvenientes se analizan riesgos como el de aplicación no homogénea de normas, o el del propio efecto del fórum shopping. Desde la perspectiva de las bondades se vislumbra desde el incremento de agentes pro competitivos y, sobretodo, el incremento de actividad tanto en enforcement como en advocacy hasta una mayor penetración potencial en la "cultura de competencia». La ocasión se presta, además, a comentar dos cuestiones de rabiosa actualidad; a saber, de un lado, la transposición de la Directiva 2014/104/UE del Parlamento Europeo y del Consejo de 26 de noviembre de 2014 relativa a determinadas normas por las que se rigen las acciones por daños en virtud del Derecho nacional, por infracciones del Derecho de la competencia de los Estados miembros y de la Unión Europea, y de otro lado, los entresijos de la economía colaborativa.

PALABRAS CLAVE: descentralización, defensa de la competència, ventajas, inconvenientes, homogénea, economia colaborativa.

${ }_{17}$ Novedosos pero equiparables a otros de naturaleza similar fruto de la ciencia y de los avances tecnológicos a lo largo del tiempo y que la propia Biblia ya nos anunciaba: $A$ la vieja economía la substituye la nueva economía. Lucas 21.

$18<$ http://acco.gencat.cat/web/.content/80_acco/documents/arxius/actuacions/ ES_7_2014_TRANSACCIONES_ENTRE_IGUALEES_Y_COMPETENCIA_CAST.pdf> 
ABSTRACT: In the present lines is addressed with necessary 'high-mindedness' both the potential inconvenients and irrefutable kindnesses of the decentralization of the Spanish system of defense of the competition. From the prism of the disadvantages there are analyzed risks as the application not homogeneous of procedure, or the effect of the forum shopping. From the perspective of the kindness it is glimpsed from the procompetitive agents' increase and, overcoat, the increase of activity so much in enforcement as in advocacy up to a major potential penetration the "culture of competition». The occasion offers the chance to comment, in addition, on two questions of highly topical, on one hand, the transposition of Directive 2014/104/UE of the European Parliament and of the Council of November 26, 2014, relative to certain procedures for which the damage actions are ruled under the National Law, by infractions of the Competition Law of the members states of the European Union and, on the other hand, the intricacies of the collaborative economy.

KEY WORDS: descentralization, defense of the competition, advantages, disadvantages, homogeneus, collaborative economy.

RECIBIDO: 18.11 .2014

ACEPTADO: 29.05.2015 